\title{
Criminologie
}

\section{Recension des dix dernières années relativement aux femmes condamnées à des peines fédérales : de mal en pis}

Kim Pate

Volume 35, numéro 2, automne 2002

Femmes et enfermement au Canada : une décennie de réformes

URI : https://id.erudit.org/iderudit/008295ar

DOI : https://doi.org/10.7202/008295ar

Aller au sommaire du numéro

Éditeur(s)

Les Presses de l'Université de Montréal

ISSN

0316-0041 (imprimé)

1492-1367 (numérique)

Découvrir la revue

Citer cet article

Pate, K. (2002). Recension des dix dernières années relativement aux femmes condamnées à des peines fédérales : de mal en pis. Criminologie, 35(2), 147-158. https://doi.org/10.7202/008295ar d'utilisation que vous pouvez consulter en ligne. 


\section{Commentaires}

\section{Recension des dix dernières années relativement aux femmes condamnées à des peines fédérales : de mal en pis ${ }^{1}$}

Kim Pate

Directrice

Association des sociétés Elizabeth Fry du Canada kpate@web.ca

Il est très difficile d'aborder ce sujet sans passer du désespoir aigu à l'indignation, et je me rends compte qu'une bonne part de mon travail et de mes gestes est dictée par des émotions qui oscillent entre ces deux sentiments. La réalité voudrait que les choses se soient grandement améliorées depuis la triste époque où il n'existait au Canada qu'une seule prison fédérale pour femmes; d'où, en partie, la difficulté de résoudre les problèmes croissants chez les femmes incarcérées dans les prisons canadiennes.

Douze années se sont écoulées depuis que le Service correctionnel du Canada (SCC) a accepté La création de choix, le rapport du groupe de travail sur les femmes condamnées à des peines fédérales, et un peu plus de six ans depuis l'ouverture de la première des nouvelles prisons et l'accusation cinglante de la juge Louise Arbour sur la façon dont le SCC gérait les châtiments. Le 6 juillet 2001, nous avons finalement célébré la fermeture de la prison pour femmes de Kingston, qui mettait un terme à ses 66 années d'incarcération de femmes et de jeunes filles.

Pour les femmes condamnées à des peines fédérales, l'ACSEF, et bien d'autres, cette célébration fut tempérée par le fait que la prison pour femmes de Kingston se voyait remplacée par dix autres unités carcérales pour femmes au sein de pénitenciers fédéraux, onze, si l'on compte le Burnaby Correctional Centre for Women, la prison provinciale pour femmes

1. Texte traduit par André Bourbonnière. 
de Colombie-Britannique. Quatre de ces centres pénitentiaires pour les femmes condamnées à des peines fédérales sont des unités à sécurité maximale isolées dans des prisons pour hommes. Pas étonnant que les femmes incarcérées demandent souvent : "Que s'est-il passé avec La création de choix?»

Après plus d'une décennie de réformes éclairées, incluant la plainte de Justice for Women devant la Commission des droits de la personne, le rapport du Comité Daubney relativement au comité permanent sur la Justice et le Solliciteur général — intitulé Taking Responsibility dans le rapport connu sous le titre La création de choix, Rapport du Groupe d'étude sur les femmes purgeant une peine fédérale - a émis une série de recommandations innovatrices. Depuis longtemps se faisaient entendre des appels à la réforme concernant le traitement des femmes incarcérées au Canada; malheureusement, dans la réalité, le système de justice criminelle est un tel géant, lourd et lent, qu'il faut lui asséner de très, très nombreuses poussées bien senties avant qu'il ne change de position.

Le groupe de travail n'était qu'une petite poussée qui prit une envergure énorme suite à la mort de sept femmes incarcérées à la prison des femmes de Kingston à la toute fin des années 1980 et au début des années 1990. Six de ces femmes étaient autochtones. Nous sommes nombreux à n'entretenir aucun doute sur le fait que c'est l'origine ethnique de ces femmes qui a amené le Service correctionnel du Canada à fournir un effort spécial pour assurer que le groupe de travail sur les femmes condamnées à des peines fédérales émette des recommandations adoptées par le Services correctionnel du Canada.

Où en sommes-nous après dix ans? D'une part, nous savons que le nombre de femmes purgeant des peines fédérales a doublé. Les femmes constituent environ $3 \%$ de la population des pénitenciers fédéraux, et 10 à $11 \%$ de celle des prisons provinciales. Il faut réaliser que, dans le monde entier, la population carcérale en plus forte croissance est celle des femmes, particulièrement des femmes jeunes, pauvres et discriminées. En fait, depuis les dix dernières années. Selon l'information provenant du Service correctionnel du Canada, au cours des deux prochaines années, nous devrions connaître une croissance de $24 \%$, et d'ici 5 ans, une croissance de $50 \%$ du nombre de femmes condamnées à des peines fédérales.

Il est important de réfléchir sur cette croissance. Les chiffres ont, à toutes fins pratiques, quintuplé dans la région de l'Atlantique et triplé dans les Prairies. Certains d'entre nous se rappelleront l'époque où il n'y avait que treize femmes en provenance des Maritimes; la dernière fois que 
j'ai parcouru la Nova Institution, j'ai recensé plus de 70 lits pour les femmes condamnées à des peines fédérales.

Dans les Prairies, la population carcérale féminine est surtout constituée de femmes autochtones, qui forment $25 \%$ de cette population. Nous savons que la croissance du nombre de femmes incarcérées est nettement liée à l'éviscération des services de santé, d'éducation et d'aide sociale; nous savons également que le cycle s'intensifie en période de ralentissement économique. Il est très facile de voir quelle direction nous faisons prendre aux gens qui subissent le pire de ces ralentissements de l'économie et des orientations sociales.

Toutefois, en termes de fréquence de condamnation, on assiste à une diminution de $7 \%$ dans l'ensemble du nombre de femmes condamnées à des peines criminelles. Cette diminution est particulièrement sensible dans le nombre de crimes violents commis par des femmes; par contre, en matière de crimes reliés à la drogue chez les femmes, on connaît une augmentation de $11 \%$. Bien que le nombre de femmes purgeant une sentence en prison ainsi que le nombre de femmes condamnées à de longues périodes d'incarcération diminue, nous assistons cependant à une augmentation du nombre de femmes purgeant de plus courtes périodes d'emprisonnement dans des pénitenciers fédéraux. Parmi les femmes incarcérées dans des pénitenciers fédéraux, $22 \%$ purgent des sentences à vie.

En tout, $82 \%$ des femmes incarcérées au fédéral purgent une première sentence de deux ans et plus, et seulement 1,6\% des femmes en sont à leur troisième incarcération fédérale ou plus. Parmi les femmes purgeant des sentences au fédéral, $4 \%$ ont été condamnées pour meurtre avec préméditation, et $14 \%$ pour meurtre sans préméditation. En ce qui a trait à la race et au racisme, environ $44 \%$ des femmes purgeant des sentences fédérales sont des femmes faisant partie de minorités ethniques, environ $25 \%$ sont autochtones, $6 \%$ noires, $1 \%$ asiatiques; les autres femmes sont décrites comme n'appartenant à aucune catégorie. À l'heure actuelle, 52\% de toutes les femmes condamnées à des peines provinciales, et $83 \%$ des femmes condamnées à des peines fédérales et présentement étiquetées comme détenues à sécurité maximale ont moins de 35 ans.

Une partie de cette augmentation est probablement due au fait que nous comptons désormais cinq pénitenciers fédéraux pour femmes, quatre unités à sécurité maximale en isolement au sein de pénitenciers pour hommes, la Isabel MacNeill Minimum Security House et le Centre correctionnel pour femmes de Burnaby, toutes ces institutions remplaçant la 
prison pour femmes de Kingston. Six de ces prisons pour femmes se trouvent dans les provinces de l'Ouest ou dans les Maritimes - deux régions qui ne possédaient auparavant aucune prison pour femmes.

On doit également garder à l'esprit que toutes ces augmentations sont survenues depuis l'accroissement des coupures budgétaires dans les services d'aide sociale, de santé et d'éducation à la grandeur du pays. Le résultat est le même ici que partout ailleurs dans le monde : ce sont les femmes qui forment le segment de croissance le plus rapide au sein de la population carcérale.

Nous savons de même qu'un nombre croissant de jeunes femmes souffrant d'incapacité d'ordre mental ou cognitif, et qui autrefois remplissaient les institutions psychiatriques ou de santé mentale, sont à l'heure actuelle de plus en plus rangées au banc des criminelles. Les tendances progressistes du passé qui visaient à désincarcérer les personnes souffrant d'incapacité cognitive ou mentale ont été bouleversées par la réduction des ressources, les attitudes et les politiques occasionnées par la démence déficitaire de la dernière décennie. Conséquence : de plus en plus de personnes sont littéralement jetées dans la rue.

Leurs tentatives pour survivre, leurs tentatives pour se médicamenter, leurs tentatives pour faire face à leur situation, tout comme le comportement qui découle du fait de se trouver dans une situation où elles sont de plus en plus privées de leurs droits, les ont conduites de plus en plus fréquemment vers la criminalité et l'emprisonnement. Une fois en prison, ces femmes considérées comme difficiles à contrôler en viendront à passer une quantité disproportionnée de leur emprisonnement sous la classification de détenues à sécurité maximale. Ce qui veut dire que, en plus de purger la plus grande part de leur sentence dans les unités de sécurité maximale isolées dans les prisons pour hommes, elles sont également très susceptibles de se retrouver en isolement. Elles tendent également à s'attirer nombre d'étiquettes psychiatriques, le Service correctionnel du Canada les caractérisant comme faisant partie des détenues les plus difficiles à contrôler par les services correctionnels canadiens.

Nombre de ces femmes portent des étiquettes telles que Syndrome d'alcoolisme fotal (SAF) ou Effet de l'alcoolisme fotal (EAF), en plus de l'étiquette de personnalité marginale. À cause de cette tendance, les femmes admises dans les prisons fédérales sont porteuses de lourds besoins. Le Service correctionnel du Canada est mal équipé pour y répondre. Comme les femmes souffrant d'incapacité cognitive ou mentale présentent un problème de contrôle pour les prisons, elles se retrouvent confinées dans les 
conditions les plus isolées, souvent en ségrégation. Ce confinement ne fait qu'exacerber les problèmes de santé mentale déjà existants. En outre, les niveaux d'isolement et la carence sensorielle qui en résulte tendent à créer des problèmes de santé mentale supplémentaires.

Singulier paradoxe, le réflexe du SCC de mettre sur pied un service de santé mentale dans les prisons ne sert qu'à aggraver la tendance à criminaliser encore plus les femmes souffrant d'incapacité cognitive ou mentale. L'établissement de tels services dans les prisons au moment où ils se raréfient dans la communauté fait en sorte que plus de femmes seront incarcérées dans des institutions fédérales parce qu'on aura présumé qu'elles pourront avoir accès en prison à des services qui ne sont pas disponibles dans la société. Les prisons ne sont et ne peuvent être des centres de traitement.

De plus, on voit la «supposée guerre aux drogues» se transformer en guerre contre les plus démunis, spécialement les femmes, puisqu'il existe un nombre croissant de femmes qui en viennent à utiliser ou à vendre de quelque façon les drogues légales ou illégales pour réussir à faire face à la vie de tous les jours ou pour leur permettre d'obtenir des ressources financières supplémentaires. On assiste également à une plus grande féminisation et criminalisation de la pauvreté.

On s'aperçoit aussi que les tendances progressives élaborées par des femmes pour combattre la violence misogyne des hommes se retournent de plus en plus contre elles. En même temps que diminue le nombre de femmes qui acceptent réellement de chercher protection auprès du système, on subit un contrecoup sous forme de politiques prétendument neutres quant au sexe et de tolérance zéro. Il en résulte que les femmes victimes de violence, dont la plupart avaient elles-mêmes appelé la police après avoir été battues, sont de plus en plus souvent contre-accusées. Cette situation est particulièrement vraie lorsque ces femmes se sont défendues contre l'abus. Dans trop de cas, les deux parties sont inculpées d'assaut, et, dans le pire des scénarios, autant l'abuseur que l'abusée se retrouvent dans les mêmes programmes de gestion de la colère.

De même, on voit croître le nombre de femmes qui, ayant exercé une force qui a provoqué la mort, plaident coupables à des accusations d'homicide ou de meurtre sans préméditation. Dans la plupart de ces cas, les femmes sont accusées de meurtre avec préméditation bien qu'elles aient réagi en légitime défense. Comme on conseille à la plupart de ces femmes de plaider coupables à des accusations de meurtre sans préméditation ou d'homicide involontaire, elles subissent également ce contrecoup. En fait, 
dans sa recension sur la légitime défense, la juge Ratushny a découvert qu'environ 20 à 30 femmes purgeant des peines fédérales relativement à la mort de conjoints violents avaient plaidé coupable; il lui devenait donc impossible de revoir leur cas.

Malgré cette sombre réalité, nous savons très bien, ceux d'entre nous qui travaillons avec les femmes détenues et qui sommes leurs alliés, qu'elles continuent à nous réclamer de tout faire pour que leur voix se fassent entendre hors des murs. C'est grâce à leur indéfectible persévérance qu'il nous est accordé le privilège de poursuivre à leur côté ce combat contestant leurs conditions de captivité au Canada.

$\mathrm{Au}$ cours des quelques prochaines années, nous prévoyons que le gouvernement canadien mettra en application d'autres politiques, réformes et initiatives régressives sous le prétexte d'endosser des décisions qui correspondent à leurs perceptions de ce qui sera acceptable pour l'opinion publique. D'autre part, l'Association canadienne des sociétés Elysabeth Fry et plusieurs autres organisations canadiennes se sont vues obligées de déposer une requête auprès de la Commission canadienne des droits de la personne concernant la discrimination et la violation des droits des femmes détenues dont fait preuve le système canadien.

Le 8 mars 2001, Journée internationale de la femme, après des années de discussions à tenter de négocier avec les services correctionnels pour qu'ils mettent en pratique toutes les recommandations communes, aussi bien que les mesures légales et les protestations officielles auprès de la Commission permanente sur la justice et les droits de la personne, l'Association canadienne des sociétés Elysabeth Fry et l'Association des femmes autochtones du Canada ont déposé une requête auprès de la Commission canadiennes des droits de la personne. Suite à ces démarches, la Commission a décidé d'entreprendre un examen dans l'ensemble du système sur la situation que subissent les femmes condamnées à des peines fédérales, s'appuyant sur son autorité en vertu de l'article 61(2) de la Loi canadienne sur les droits de la personne pour rédiger un compte rendu sur la façon dont le gouvernement du Canada exerce de la discrimination envers les femmes purgeant des peines de deux ans ou plus.

Nous alléguons que la violation de leurs droits humains est causée par la discrimination sur la base du sexe, de la race et de l'incapacité. Dans les années 1980, un groupe qui se présentait sous le nom de Justice for Women, et Gayle Horii, une femme purgeant une sentence à vie, déposèrent une plainte auprès de la Comission canadienne des droits de la personne reposant substantiellement sur les mêmes préoccupations que celles que 
nous soulevons. Au moment du dépôt des premières plaintes, il n'existait encore qu'un seul pénitencier fédéral pour femmes, où les besoins des femmes étaient négligés; en comparaison, les hommes avaient accès à beaucoup plus de programmes et de services. Les plaintes de Gayle Horii auprès de la Commission canadienne des droits de la personne ainsi que le procès en cours datent maintenant d'environ quinze ans.

La plainte de l'Association canadienne des sociétés Elysabeth Fry expose, entre autres questions, que, contrairement à ce qui se passe chez les hommes, exception faite de treize lits réservés aux femmes en fin de sentence, les femmes qui sont classifiées comme détenues à sécurité minimale n'ont pas accès aux prisons à sécurité minimale. Qui plus est, malgré les promesses de La création de choix et de la Loi sur le système correctionnel et la mise en liberté sous condition, il n'existe pas assez d'options de réintégration dans la société pour les femmes, spécialement les femmes autochtones. De la même façon, en plus d'être assujetties à un procédé de classification discriminatoire, les femmes classifiées comme détenues à sécurité maximale et celles qui sont identifiées comme souffrant d'incapacité d'ordre mental ou cognitif ne jouissent pas de conditions d'incarcération adéquates.

Les femmes autochtones composent 1 à $2 \%$ de la population générale, mais forment $24 \%$ de la population des femmes condamnées à des peines fédérales. De ces femmes vivant en particulier dans les Prairies, environ la moitié languissent dans des unités à sécurité maximale isolées dans des prisons pour hommes. Les femmes identifiées comme souffrant d'incapacité d'ordre mental ou cognitif sont confrontées à une situation semblable. En plus d'être soumises à un système qui classifie un trop grand nombre d'entre elles comme risque à sécurité maximale, ces femmes sont de plus en plus isolées dans ce que l'Enquêteur correctionnel qualifie d'environnement brutal et discriminatoire.

Pire encore, bien que le Service correctionnel du Canada et le Solliciteur général se soient engagés à fermer les unités à sécurité maximale isolées dans des prisons pour hommes vers la fin de septembre de cette année, le Service correctionnel du Canada prévoit reproduire ces unités d'isolation dans les nouvelles prisons régionales. Non seulement les nouvelles unités à sécurité maximale vont-elles reproduire l'isolement des unités de ségrégation, sans aucun respect des droits réservés aux prisonnières isolées selon la loi canadienne, mais en plus le SCC prévoit incorporer des postes protégés pour les officiers au sein de ces unités.

Également, le SCC a établi un nouveau «protocole de gestion» pour les femmes classifiées prisonnières à sécurité maximale, ce qui entrainera la vio- 
lation, entérinée par le SCC, de la Charte et des droits de la personne des femmes, sans mentionner les droits et les protections formulés dans la Loi sur le système correctionnel. En plus d'être contraires aux principes de justice fondamentale, ces réalités ne vont servir qu'à accroître cet isolement des femmes qui nous inquiète déjà en raison des dommages évidents et démontrés, en plus du danger infligé aux femmes prisonnières.

Les membres du Groupe de travail sur les femmes condamnées à des peines fédérales s'inquiétaient beaucoup, entre autres, que leur travail n'aboutisse à la création de cinq mini-prisons pour femmes. Le nouveau protocole de gestion et les plans pour des unités sécuritaires pour les femmes classifiées prisonnières à sécurité maximale dans les prisons régionales constituent de claires indications que, pour beaucoup trop de femmes, les nouvelles prisons évoluent de mal en pis.

La plainte de l'ACSEF relative aux droits de la personne est aussi appuyée par le Réseau d'action des femmes autochtones (Aboriginal Women's Action Network), l'Assemblée des Premières Nations, l'Association nationale des centres de rencontre, la Federation of Saskatchewan Indian Nations, Strength in SISterhood, le Réseau des femmes handicapées du Canada, le Comité national d'action sur la condition de la femme, la National Association of Women and the Law, l'Association canadienne des centres contre le viol, l'Institut canadien de recherches sur les femmes, l'Association du Barreau canadien, et Amnistie Internationale, par de nombreux députés locaux de même que par des membres individuels de l'Association canadienne des sociétés Elizabeth Fry. Human Rights and Prison Watch International de même que Amnistie Internationale ont déjà exprimé leur inquiétude concernant les abus des droits de la personne dans les prisons canadiennes pour femmes.

Le Canada est fier de sa réputation internationale relativement aux droits de la personne. Quand il s'agit de la façon dont nous traitons nos éléments les plus marginalisés, cette réputation est trop souvent non fondée. Le gouvernement canadien refuse de mettre en pratique des recommandations répétées par le Groupe d'étude sur les femmes purgeant une sentence fédérale et l'isolement du Service correctionnel du Canada visant une surveillance externe, et de suivre les recommandations de sa propre commission quant à une surveillance judiciaire. Le SCC a même rejeté les recommandations du Comité permanent du Parlement sur la justice et les droits de la personne sur ce point.

Nous espérons que la Commission canadienne des droits de la personne va se pencher sur les problèmes du système qui, pour les femmes 
condamnées à des peines fédérales, contribuent à un contexte correctionnel dont le Canada doit être tenu responsable. Les abus aux droits de la personne subis par les femmes condamnées à des peines fédérales constituent une situation honteuse pour tous les Canadiens.

À ceux qui s'intéressent au sort des femmes incarcérées, Pat Carlen et bien d'autres personnes ne cessent de réclamer de mettre fin aux demandes de réformes sans avenir pour exiger la désincarcération des femmes et l'abolition des prisons pour femmes. S'appuyant en grande partie sur les résultats de l'expérience du Groupe de travail de 1993, l'ACSEF a, elle aussi, adopté cette position. En conséquence, nous poursuivons notre bataille pour l'égalité des femmes en continuant notre travail en faveur des femmes incarcérées et avec elles.

Dans cette optique visant l'abolition de l'incarcération des femmes, nous avons mis en place des coalitions en vue de riposter au programme régressif d'ordre public et à l'incarcération des femmes. L'an dernier, nous avons mis l'accent sur cette coalition lors d'une incroyable conférence de femmes que nous avons commanditée et organisée conjointement avec l'Association canadienne des centres contre le viol (ACCCV).

Depuis environ dix ans, l'ACSEF et l'ACCCV travaillent ensemble sur nombre de problèmes importants concernant l'égalité des femmes. Ces deux organismes, de concert avec des femmes qui ont connu la victimisation et la criminalisation, avec certains autres groupes militant pour l'égalité et représentant des femmes handicapées, des groupes autochtones et d'autres groupes victimes de préjugés raciaux, de même également qu'avec des universitaires, des professionnels et praticiens, se sont tous unis dans leurs efforts pour réformer politiques et lois.

La conférence, intitulée Women's Resistance : From Victimization to Criminalization, a été une occasion sans précédent pour les femmes incarcérées ou provenant des prisons, des centres d'aide aux victimes d'agression sexuelle ou des maisons de refuge pour femmes battues, pour les travailleuses antiprison et antiviolence de première ligne, avocates, universitaires, politiciennes, bureaucrates et autres professionnelles, de se réunir afin d'élaborer des stratégies pour favoriser l'égalité des femmes.

Contrairement à ce qui se passe généralement, les plus démunies ont été privilégiées par rapport aux professionnelles et aux universitaires lors de la conférence Women's Resistance. Du reste, toutes les participantes ont émis des commentaires très positifs sur la valeur et la qualité des comités et des ateliers de travail, ainsi que sur les interactions générales et l'implication de toutes les participantes. Au niveau local ou populaire, la 
conférence a inspiré nombre de nouveaux partenariats et d'activités. L'ACSEF et l'ACCCV considèrent comme une preuve du succès de la conférence le grand nombre de nouveaux projets qui ont surgi à travers le pays. Par exemple, on constate une plus grande ouverture des travailleurs antiviolence à œuvrer avec les femmes criminalisées. De plus, des démarches ont été entreprises vers l'unification d'une position antiprostitution chez les travailleuses antiviolence du Québec dans le contexte actuel du travail de la Fédération des femmes du Québec (FFQ).

En outre, la conférence a invité les critiques des systèmes de maintien de l'ordre, des cours et des services de correction, quelle que soit leur position dans la hiérarchie, à amorcer un dialogue productif servant à élever le niveau général de sensibilisation et à inspirer de nouveaux partenariats originaux pour contester le statu quo. Les participantes à la conférence ont vigoureusement témoigné leur intérêt et leur accord pour former des partenariats et des coalitions visant à surveiller et à critiquer le comportement de la force policière à l'échelle du pays. L'insatisfaction était aussi généralisée relativement aux forces de l'ordre dans la communauté, ce qui a amené la mise en place de mécanismes de surveillance pour détecter la réponse policière à la violence faite aux femmes.

Les femmes ordinaires ont appris que les restrictions appliquées à leurs salaires, à leurs conditions de travail et à leurs dépenses d'exploitation ne relèvent pas tant d'une prise de décision de structure, mais d'une prise de décision des gouvernements fédéral et provincial. Beaucoup de travailleuses de première ligne ont aussi pris connaissance de la faible marge économique qui les sépare des femmes pour lesquelles elles œuvrent, comparativement à l'écart qui existe entre elles et les femmes professionnelles. En grand nombre, les travailleuses de première ligne ainsi que les femmes criminalisées et victimisées ont amené les femmes professionnelles à examiner la nature particulière de leur privilège face à l'expertise toute spéciale des femmes dépourvues de ce privilège sur les questions de loi, tout spécialement en ce qui concerne les cas de violence.

Suite à la conférence Women's Resistance, les participantes ont acquis une meilleure perspective des relations entre victimisation, prostitution, criminalisation et incapacité d'ordre mental ou cognitif chez les femmes et les jeunes filles. Certaines de ces femmes ont décidé d'entreprendre des activités conçues pour aborder ces relations; plus particulièrement, certaines femmes travaillent à élaborer des stratégies pour répondre au besoin identifié de stratégies d'adaptation pouvant aider ces femmes à 
s'organiser pour trouver des solutions à la dépossession et à l'oppression qu'elles subissent.

Autant l'ACSEF que l'ACCCV travaillent à élaborer des stratégies nationales pour s'assurer que les services financés et gérés par la province n'entravent pas la Charte et les garanties des droits de la personne; elles veillent aussi à isoler le travail de nos membres des pressions du provincial voulant que nous abandonnions le côté égalitaire de notre travail en faveur de la simple fourniture de services. Ces stratégies vont aussi servir à réagir à la décharge de responsabilité des paliers provincial et municipal sans les ressources nécessaires pour protéger les plus démunies et les plus vulnérables. Les deux organisations ont identifié des groupes de travail clés aux niveaux fédéral, provincial et territorial comme étant des domaines essentiels d'engagement.

En outre, nombre de ces participantes ont intégré les rangs de notre coalition croissante de femmes, tant au Canada que dans le reste du monde, qui œuvrent à l'abolition du recours à l'incarcération pour les femmes. Cette coalition a pris encore plus d'importance avec l'arrivée d'Angela Davis et d'autres femmes impliquées dans le mouvement de résistance critique opposé au «complexe industriel de prisons» américain, et de femmes de la conférence Sisters Inside qui s'est déroulée en Australie, en novembre 2001. L'engagement de femmes travaillant en pays occidentaux ou dans des pays en voie de développement continue à se renforcer et à croître à mesure que nous joignons nos forces pour établir des stratégies viables de décarcération.

Gayle Horii, condamnée à perpétuité, me répète souvent ces paroles : «Femmes unies, femmes fortes». Lors de notre première rencontre en 1992, Gayle m'avait cité ces mots de Lilla Watson, une autochtone australienne, et qui incarnent et transmettent le mieux le message de notre travail :

Si tu es venue ici pour m'aider,

tu perds ton temps.

Si tu es ici parce que

ta libération est liée à la mienne, alors travaillons ensemble. 


\section{Références}

Arbour, L. (1996). Commission d'enquête sur certains événements à la Prison pour femmes de Kingston. Ottawa : Public Works and Government Services of Canada.

ACSEF et ACCCV. (2002). Rapport sur la conférence de l'ACSEF - CASAC Women's Resistance: From Victimization to Criminalization. Ottawa.

Carlen, P. Sledgehammer. Don't have resources with me so going from memory bere....

Comité permanent sur la Justice et le Solliciteur général (1988). Taking Responsibility. Ottawa.

Renseignements statistiques provenant d'une mise à jour de l'article sur les femmes délinquantes, Service correctionnel du Canada, novembre 2001.

Groupe de travail sur les femmes condamnées à des peines fédérales (1990). Creating choices. Ottawa : Ministère du Soliciteur Général. 